\title{
NON-OBSTRUCTED SUBCANONICAL SPACE CURVES
}

\author{
Rosa M. Miró-RoIG*
}

To Pere

\begin{abstract}
Recall that a closed subscheme $X \subset \mathbf{P}$ is non-obstructed if the corresponding point, $x$ of the Hilbert scheme $H_{i l b}{ }_{p(t)}^{n}$ is non-singular. A geometric characterization of non-obstructedness is not known even for smooth space curves. The goal of this work is to prove that subcanonical $k$-Buchsbaum, $k \leq 2$, space curves are nonobstructed. As a main tool, wo use Serre's correspondence between subcanonical curves and vector bundles.
\end{abstract}

\section{Introduction}

In 1960 ([G]), A. Grothendieck proved that there is a $k$-projective scheme $\mathrm{Hilb}_{p(t)}^{n}$ which paramelrizes from the functorial point of view all closed subschemes of $\mathbf{P}^{n}$ with given Hilbert polynomial $p(t) \in \mathbf{Q}[t]$; though so far very few of these schernes has been studied in detail and there are no general results about these schemes concerning connected components, dimension, smoothness, rationality, topological invariants,... From now on, we will say that a closed subscheme $X \subset \mathbf{P}^{n}$ is non-obstructed if the corresponding point $x$ of the Hilbert scheme Hilb ${ }_{p(t)}^{n}$ is non-singular; otherwise, we will say that $X$ is obstructed. A geonetrical characterization of non-obstructedness is not known even for smooth space curves and scveral examples of obstructed smooth space curves has been given, for instance, in $[\mathbf{M}]$, [S], [EF], [K1], [K2], [K3], [E1], and [BKM].

In this pajer, we will prove the non-obstructedness of subcanonical 2-Buchsbaum space curves (CF. Theorem 2.5). As a corollary we will get that 2-Buchsbaum quasi-complete space curves (Cf. Definition 2.6)

*Partially suported by DGiCY'T PB88-0224. 
not contained in a surface of degree $<9$ are also non-obstructed (Cf. Proposition 2.7).

Recall that a curve $C \subset \mathbf{P}^{3}$ is subcanonical if the canonical sheaf $w_{C}$ of $C$ is isomorphic to $\mathcal{O}_{C}(a)$ for some integer $a$; and a curve $C \subset \mathbf{P}^{3}$ is $k$-Buchsbaum if and only if $k=\min \left\{t \mid m^{l} M(C)=0\right\}$ where $m=\left(X_{0}, X_{1}, X_{2}, X_{3}\right)$ and $M(C)$ is the Hartshorne-Rao module of $C$. Note that a curve $C \subset \mathbf{P}^{3}$ is arithmetically Cohen-Macaulay (Resp. arithmetically Buchsbaum) if and only if is 0-Buchsbaum (Resp. 1Buchsbaum). So, the notion of $k$-Buchsbaum can be viewed as a natural cxtension of the notions arithmetically Cohen-Macaulay and arithmetically Buchsbaum. Moreover, every curve $C \subset \mathbf{P}^{3}$ is $k$-Buchsbaum for some integer $k$.

A classical theorem of Gherardeli says that a smooth irreducible subcanonical curve $C \subset \mathbf{P}^{3}$ is arithmetically Cohen-Macaulay if and only if it is cornplete intersection (For a weaker characterization of complete intersection space curves see $[\mathbf{C V}]$ ). In $[\mathbf{E F}]$, Ellia-Fiorentini prove that an integral subcanonical curve $C \subset \mathbf{P}^{3}$ is arithmetically Buchsbaum if and only if $C$ is the zero scheme of a section of $N(l), t \geq 1$, where $N$ is the null correlation bundle. Therefore, subcanonical $k$-Buchsbeum space curves, $0 \leq k \leq 1$, are non-obstructed. The aim of this paper is to extend this knowledge to subcanonical, 2-Buchsbaum space curves with the hope of finding a clue which could facilitate the study of arbitrary subcanonical space curves.

In section 1, we establish some preliminary results. In section 2, we prove the main results of this paper. We see that any subcarionical, 2Buchsbaum space curve is in the even liaison class of three disjoint lines. From this we can show that any subcanonical, 2-Buchsbaum space curve is non-obstructed, it has maximal rank and we give a resolution of its ideal sheal. In section 3, we conclude by studying some examples and adding some remarks.

\section{Notations}

Throughout this paper we work over an algebraically closed field $\mathbf{k}$ of characteristic zero. We set $S=\mathrm{k}\left[X_{0}, \ldots, X_{3}\right], m=\left(X_{0}, \ldots, X_{3}\right) \subset S$ and $\mathbf{P}^{3}=\operatorname{Proj}(S)$. By a curve we mean a closed, locally CohenMacaulay, one-dimensional subscheme $X \subset \mathbf{P}^{3}$. For a coherent sheaf $F$ on $X, F(n)$ as usual will be $F \otimes \mathcal{O}_{X}(n)$ and we let $h^{i} F(n)=$ $\operatorname{dim}_{\mathrm{k}} H^{i}(X, F(n))$.

Giver a curve $C \subset \mathbf{P}^{3}$, we denote $d=$ degree of $C, p_{a}=$ arithmetic genus of $C, s=\min \left\{t: H^{0} I_{C}(t) \neq 0\right\}, e=\operatorname{mix}\left\{t: H^{1} \mathcal{O}_{C}(t) \neq 0\right\}$ 
and $c=\max \left\{t \mid H^{1} I_{C}(t) \neq 0\right\}(c=-\infty$, if $C$ is arithmetically CohenMacaulay). For a curve $C$ in $\mathrm{P}^{3}$, the Hartshorne-Rao module $M(C)=$ $\oplus_{n} H^{1} I_{C}(n)$ is a graded $S$-module of finite length. Recall that a curve $C$ in $\mathrm{P}^{3}$ is said to be a-subcanonical if the canonical sheaf $\omega_{C}$ of $C$ is isomorphic to $\mathcal{O}_{C}(a)$ and a curve $C$ in $\mathrm{P}^{3}$ is said to have rnaximal rank if the restriction map $H^{0}\left(\mathbf{P}^{3}, \mathcal{O}_{\mathrm{P}^{3}}(n)\right) \rightarrow H^{0}\left(C, \mathcal{O}_{C}(n)\right)$ is of maximal rank, for every integer $n$.

For a colierent sheaf $E$ on $\mathbf{P}^{3}$ we denote $H_{*}^{1}(E)$ the graded $S$-module $\oplus_{n} H^{1}\left(\mathbf{P}^{3}, E(n)\right) . \Lambda$ rank 2 vector bundle $E$ on $\mathbf{P}^{3}$ is said to be stable if $H^{0} E_{\text {norm }}=0$ where $E_{\text {norm }}$ denotes the twist of $E$ which has first Chern class equal 0 or -1 . Our main reference for this subject is $[\mathbf{H}]$.

\section{Generalities}

In the present section we recall the definitions and basic propertics needed later.

Definition 1.1. ([E], $[\mathrm{MM}])$ Let $C \subset \mathbf{P}^{3}$ be a curve. We say that $C$ is $k$-Buchsbaum if and only if $k=\min \left\{t \mid m^{t} M(C)=0\right\}$.

See $[\mathrm{MM}]$ for general results on $k$-Buchsbaum curves.

Remarks 1.1.1. (a) $C$ is 0 -Buchsbaum (Resp. 1-Buchsbaum) if and only if $C$ is arithmetically Colen-Macaulay (Rosp. arithmetically Buchsbaum).

(b) For any curve $C \subset \mathbf{P}^{3}$ there is an integer $k$ such that $C$ is $k$ Buchsbaum.

(c) Let $C, D \subset \mathbf{P}^{3}$ be two curves in the same liaison class. Then, $C$ is $k$-Buchsbaum if and only if $D$ is $k$-Buchsbaum.

Definition 1.2. ([E], $[\mathbf{M M}]$ ) A rank 2 vector bundle $E$ on $\mathbf{P}^{3}$ is said to be $k$-Buclisbaum if and only if $k=\min \left\{t \mid m^{t} H_{*}^{1} E=0\right\}$.

Remark 1.2.1. If $E$ is a rark 2, $k$-Buchsbaum vector bundle on $\mathbf{P}^{3}$, then the zero set of a section of $E(n)$ is a $k$-Buchsbaum, subcanonical curve. Cornversely, any subcanonical, $k$-Buchsbaum curve corresponds to a rank $2, k$-Buchsbaum vector bundle on $\mathbf{P}^{3}$.

From $[\mathbf{E}]$, we get the following characterization for rank $2, k$ Buchsbaum, $k \leq 2$, vector bundles on $\mathbf{P}^{3}$.

Proposition 1.3. Let $E$ be a normalized, rank 2, $k$-Buchsbaurn vector bundle on $\mathbf{P}^{3}$. Then:

$k=0$ if and only if $E$ is direct sum of line bundles,

$k=1$ if and only if $E$ is the a correlation bundle,

$k=2$ if and only if $E$ is stable, $c_{1} E=0$ and $c_{2} E=2$. 
Proposition 1.4. Let $E$ be a rank 2 stable vector bundle on $\mathbf{P}^{3}$ with $c_{1} E=0, c_{2} E=2$. Then:

(1) $h^{3} E(t)=0$ for $t \geq-4, h^{2} E(t)=0$ for $t \neq-3,-4$ and $h^{2} E(t)=2$ for $t=-3,-4, h^{1} E(t)=0$ for $t \neq-1,0$ and $h^{1} E(t)=2$ for $t=0,-1, h^{0} E(t)=0$ for $t \leq 0$ and $h^{0} E(t)=\chi E(t)$ for $t>0$, and $E(2)$ is generated by its global scctions,

(2) There is a section $s \in H^{0} E(1)$ whose zero set is three disjoint lines,

(3) E has a locally free resolution of the following kind:

$$
0 \longrightarrow 2 \mathcal{O}(-4) \longrightarrow 6 \mathcal{O}(-3) \longrightarrow 2 \mathcal{O}(-1) \oplus 4 \mathcal{O}(-2) \longrightarrow E \longrightarrow 0
$$

Furthermore, the moduli space $M(0,2)$ of rank 2 stable vector bundles on $\mathbf{P}^{3}$ with $c_{1}=0, c_{2}=2$ is an irreducible, smooth variety of dimension 13.

Proof: I will only proof (3); for the other results see [H]. By (2), there is a section $s \in H^{0} E(1)$ which gives us an exact sequence:

$$
0 \longrightarrow \mathcal{O} \longrightarrow E(1) \longrightarrow I_{Y}(2) \longrightarrow 0
$$

where $Y$ is the disjoint union of three lines.

By [I, Proposition 7.2.2], $I_{Y}$ has a resolution of the following kind:

$$
0 \longrightarrow 2 \mathcal{O}(-5) \longrightarrow 6 \mathcal{O}(-4) \longrightarrow \mathcal{O}(-2) \oplus 4 \mathcal{O}(-3) \longrightarrow \Gamma_{Y} \longrightarrow 0
$$

Set $K:=\operatorname{Ker}\left(4 \mathcal{O}(-3) \oplus \mathcal{O}(-2) \rightarrow I_{Y}\right)$. Then, the exact sequence (2) breaks up and gives us the exact sequences:

$$
\begin{aligned}
& 0 \longrightarrow 2 \mathcal{O}(-5) \longrightarrow 6 \mathcal{O}(-4) \longrightarrow K \longrightarrow 0 \\
& 0 \longrightarrow K \longrightarrow \mathcal{O}(-2) \oplus 4 \mathcal{O}(-3) \longrightarrow I_{Y} \longrightarrow 0 .
\end{aligned}
$$


The exact sequences (1) and (4) give us the following diagram:
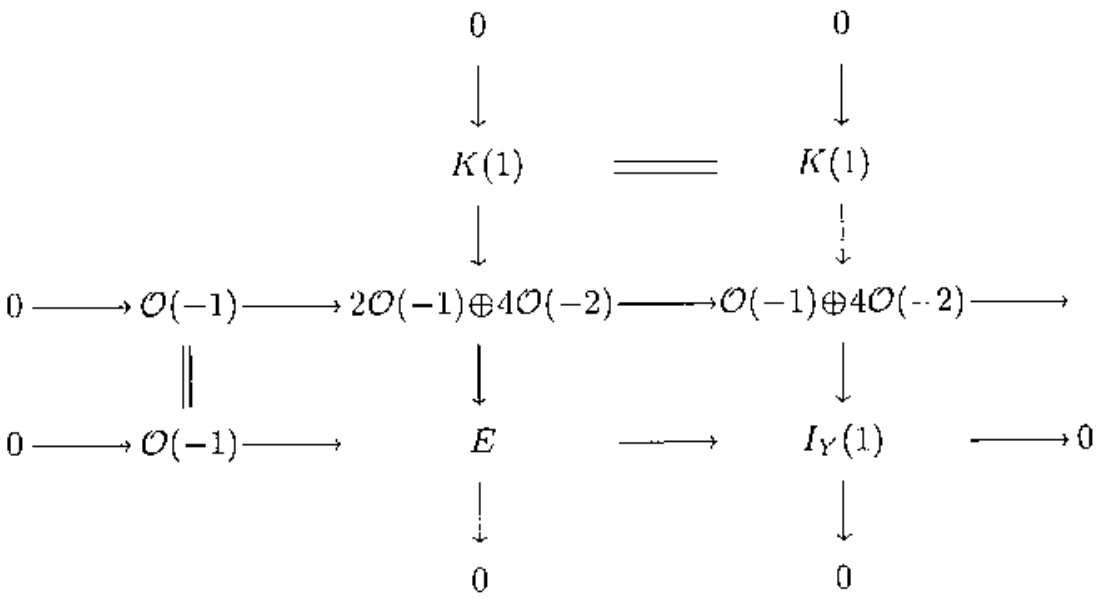

Finally, the exact sequence (3) together with the exact sequence:

$$
0 \longrightarrow K(1) \longrightarrow 2 \mathcal{O}(-1) \oplus 4 \mathcal{O}(-2) \longrightarrow E \longrightarrow 0
$$

gives us the resolution of $E$ :

$0 \longrightarrow 2 \mathrm{O}(-4) \longrightarrow 6 \mathrm{O}(-3) \longrightarrow 2 \mathrm{O}(-1) \mathrm{O} 4 \mathrm{O}(-2) \longrightarrow E \rightarrow 0$.

\section{Subcanonical, 2-Buchsbaum space curves}

In this section, we give a complete description of subcanonical, 2 Buchsbaum space curves.

Theorem 2.1. Let $C \subset \mathrm{P}^{3}$ be an integral, a-sabcanonucal curve. Then, $C$ is a $-B$ uchsbaum if and only if $C$ is the zero scheme of a section of a rank 2 stable vector bundle $E$ on $\mathbf{P}^{3}$ with $c_{1} E=0$ and $c_{2} E=2$.

Proof: Jet $E$ be a rank 2 stable vector bundle on $\mathrm{P}^{3}$ with $c_{1} E=0$ and $c_{2} E=2$. By Proposition $1.3, E$ is 2-Buchsbaum. In particular: any curve associated to $E$ is 2 -Buchsbaum.

Conversely, let, $C \subset \mathbf{P}^{3}$ be an integral, 2-Buchsbaum, $a$-subcrnonical curve. Set $b=a / 2$ if $a$ is cven, and $b=(a+1) / 2$ if $a$ is odkl. A general section $0 \neq s \in H^{(0} \omega_{C} \simeq H^{0} \mathcal{O}_{C}(a)$ gives us an cxact sequence

$$
0 \longrightarrow \mathcal{O} \longrightarrow E(b+2) \longrightarrow I_{C}(a+4) \longrightarrow 0
$$

where $E$ is a rank 2 vector bundle on $\mathrm{P}^{3}$. Since a 2 -Buchsbaum curve gives risc to a 2-Buchsbanm sheaf, applying Proposition 1.3, we get that, $E$ is stable, $c_{1} E=0$ and $c_{2} E=2$. 
Proposition 2.2. Let $F$ be a rank 2 stable vector bundle on $\mathbf{P}^{3}$ with $c_{1}=0, c_{2}=2$ and let $C \subset \mathbf{P}^{3}$ be the zero scheme of a section of $E(t)$, $t \geq 1$. Then:

(1) $\omega_{C} \simeq \mathcal{O}_{C}(2 t-4), e(C)=2 t-4, s(C)=t+1, \operatorname{deg}(C)=t^{2}+2$, $p_{a}=t^{3}-2 t^{2}+2 t-3$

(2) $M(C)_{t-1} \simeq M(C)_{t} \simeq \mathrm{k}^{2}, M(C)_{n}=0$ for $n \neq t, t-1$,

(3) $C$ has maximal rank and belongs to the even liaison class of three disjoint lines,

(1) The ideal sheaf $I_{C}$ of $C$ has a locally free resolution of the following kind:

$$
\begin{aligned}
& 0 \longrightarrow 2 \mathcal{O}(-5) \longrightarrow 6 \mathcal{O}(-4) \longrightarrow 4 \mathcal{O}(-3) \oplus \mathcal{O}(-2) \longrightarrow I_{C} \longrightarrow 0 \\
& \text { if } t=1 \text {, and } \\
& 0 \longrightarrow 2 \mathcal{O}(-4-t) \longrightarrow 6 \mathcal{O}(-3-t) \oplus \mathcal{O}(-2 t) \longrightarrow \\
& \longrightarrow 4 \mathcal{O}(-2-t) \oplus 2 \mathcal{O}(-1-t) \rightarrow I_{C} \longrightarrow 0 \\
& \text { if } t>1 \text {. }
\end{aligned}
$$

Proof: (1)-(3) follows from Proposition 1.4 and the exact sequence

$$
0 \longrightarrow \mathcal{O} \longrightarrow E(t) \longrightarrow I_{C}(2 t) \longrightarrow 0
$$

(4) If $t=1$, then $C$ is the disjoint union of three lines and the result follows from [I, Proposition 7.2.2].

Assume $t>1$ and consider the locally free resolution of $E$ given in Proposition 1.4:

$$
0 \longrightarrow 2 \mathcal{O}(-4) \longrightarrow 6 \mathcal{O}(-3) \longrightarrow 4 \mathcal{O}(-2) \oplus 2 \mathcal{O}(-1) \longrightarrow E \longrightarrow 0
$$

Set $K:=\operatorname{Ker}(4 \mathcal{O}(-2) \oplus 2 \mathcal{O}(-1) \rightarrow E)$. Then, the exact sequence (1) breaks up and gives tis the exact sequences:

$$
\begin{aligned}
& 0 \longrightarrow 2 \mathcal{O}(-4) \longrightarrow 6 \mathcal{O}(-3) \longrightarrow K \longrightarrow 0 \\
& 0 \longrightarrow K \longrightarrow 4 \mathcal{O}(-2) \oplus 2 \mathcal{O}(-1) \longrightarrow E \longrightarrow 0
\end{aligned}
$$

The exact sequence (3) together with the exact sequence:

$$
0 \longrightarrow \mathcal{O}(-2 t) \longrightarrow E(-t) \longrightarrow I_{C} \longrightarrow 0
$$


gives us the following diagram:

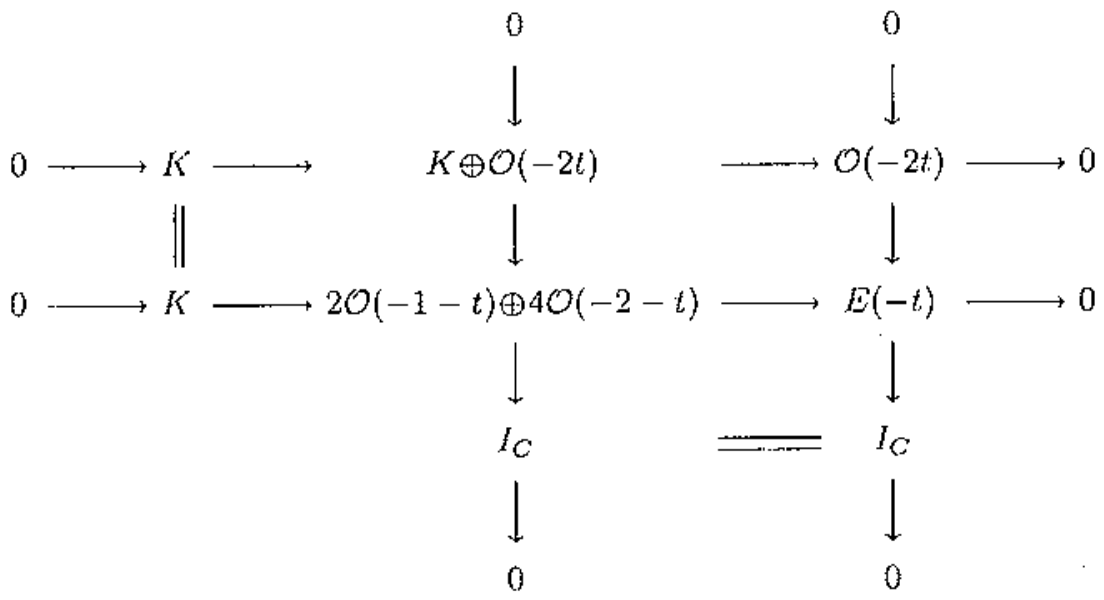

Finally, using the exact sequence (2) and the exact sequence:

$$
0 \longrightarrow K \oplus \mathcal{O}(-2 t) \longrightarrow 2 \mathcal{O}(-1-t) \oplus 4 \mathcal{O}(-2-t) \longrightarrow I_{C} \longrightarrow 0
$$

we get the locally free resolution of $I_{C}$ :

$$
\begin{aligned}
0 \longrightarrow 2 \mathrm{O}(-4-t) \longrightarrow & 6 \mathcal{O}(-3-t) \oplus \mathcal{O}(-2 t) \longrightarrow \\
& \longrightarrow 4 \mathcal{O}(-2-t) \oplus 2 \mathcal{O}(-1-t) \longrightarrow I_{C} \longrightarrow 0 .
\end{aligned}
$$

Lemma 2.3. Let $E$ be a rank 2 stable vector bundle on $\mathrm{P}^{3}$ with $c_{1} E=$ $0, c_{2} E=2$. Then, $h^{2} E \otimes E=h^{3} E \otimes E=0$.

Proof: By proposition 1.4, $E$ has a locally free resolution of the following kind:

(1) $0 \longrightarrow 2 \mathrm{O}(-4) \longrightarrow 6 \mathrm{O}(-3) \longrightarrow 4 \mathcal{O}(-2) \oplus 2 \mathcal{O}(-1) \longrightarrow E \longrightarrow 0$.

Set $K:=\operatorname{Ker}(4 \mathcal{O}(-2) \oplus 2 \mathcal{O}(-1) \rightarrow E)$. Then, the exact sequence (1) breaks up and gives us the exact sequences:

$$
\begin{aligned}
& 0 \longrightarrow 2 \mathrm{O}(-4) \longrightarrow 6 \mathrm{O}(-3) \longrightarrow K \longrightarrow 0 \\
& 0 \longrightarrow K \longrightarrow 4 \mathcal{O}(-2) \oplus 2 \mathrm{O}(-1) \longrightarrow E \rightarrow 0 .
\end{aligned}
$$

Finally, tensoring (2) and (3) by $E$ and taking cohomology we get $h^{2} E \otimes E=h^{3} E \otimes E=0$.

Let $\mathcal{F}(t)$ be the fanily of curves zero schemes of sections of $E(t)$, $t \geq 1$, where $E$ is a rank 2 stable vector bundle on $\mathrm{P}^{3}$ with $c_{1} E=0$ and $c_{2} E=2$. 
Proposition 2.4. (1) For all $t \geq 1, \mathcal{F}(t)$ is an irreducible family of dimension 12 if $t=1$ and $2 t^{2}+10+\left(t^{3}+5 t\right) / 3$ if $t>1$.

(2) For $t \neq 3,4$, the closure of $\mathcal{F}(t)$ in Hilb $\mathrm{P}^{3}$ is an irreducible component generically smooth.

(3) $\mathcal{F}(3)$ (Resp. $\mathcal{F}(4)$ ) is contained in a unique component of $\mathrm{Hilb} \mathrm{P}^{3}$, generically smooth of dimension 44 (Resp. 72).

Proof: (1) The irreducibility of $\mathcal{F}(t)$ follows from the irreducibility of $M(0,2)$. On the other hand, $\operatorname{dim} \mathcal{F}(t)=\operatorname{dim} M(0,2)+h^{0} E(t)-h^{0} \mathcal{O}_{C}$ for a general $E$ in $M(0,2)$ and a general $C$ in $\mathcal{F}(t)$. So, the dimension of $\mathcal{F}(t)$ is 12 if $\mathrm{t}=\mathrm{I}$ and $2 t^{2}+10 t+\left(t^{3}+5 t\right) / 3$ if $t>1$.

(2) Assume $t \neq 3,4$. Let $C$ be a curve in $\mathcal{F}(t)$ and let $N_{C}$ be its normal bundle. Since the Zariski tangent space of Hilb $\mathrm{P}^{3}$ at the point corresponding to $C$ is isomorphic to $H^{\circ} N_{C}$ and $h^{0} N_{C}=4 \operatorname{deg}(C)+$ $h^{1} N_{C}$, it is enough to compute $h^{1} N_{C}$ and to check that $\operatorname{dim} \mathcal{F}(t)=$ $h^{0} N_{C}$. But, $C$ is the zero scheme of a section of $E(t)$, hence $N_{C} \simeq$ $E(t) \otimes \mathcal{O}_{C}$ and we have $h^{1} N_{C}=h^{1}\left(E(t) \otimes \mathcal{O}_{C}\right)=h^{2}\left(E(t) \otimes I_{C}\right)$. On the other hand, tensoring by $E(t)$ the exact sequence:

$$
0 \longrightarrow \mathcal{O}(-2 t) \longrightarrow E(-t) \longrightarrow I_{C} \longrightarrow 0
$$

taking cohomology and using lemma 2.3, we get:

$$
h^{2}\left(E(t) \otimes I_{C}\right)= \begin{cases}0 & \text { if } t=1,2 \\ 2-2 t^{2}+\left(t^{3}+5 t\right) / 3 & \text { if } t>4\end{cases}
$$

Putting altogether we have: for $t=1,2, \operatorname{dim} \mathcal{F}(t)=h^{0} N_{C}=4 \operatorname{deg}(C)$ and $h^{1} N_{C}=0$ and for $t>4, \operatorname{dim} \mathcal{F}(t)=h^{0} N_{C}$; which gives what we want.

(3) Assume $t=3,4$. Let $C$ be a curve of $\mathcal{F}(3)$ (Resp. $\mathcal{F}(4)$ ) and let $N_{C}$ its normal bundle. As before, we compute $h^{1} N_{C}$ and we get: $h^{1} N_{C}=0$ and $\operatorname{dim} \mathcal{F}(t)<4 \operatorname{deg}(C)=h^{0} N_{C}=44$ (Resp. 72). So, $\mathcal{F}(3)$ (Resp. $\mathcal{F}(4)$ ) is contained in a unique irreducible component of Hilb $\mathbf{P}^{3}$, generically smooth of dimension 44 (Resp. 72).

Theorem 2.5. Let $C \subset P^{3}$ be an irreducible, subcanonical curve. If $C$ is $k$-Buchsbaum and $k \leq 2$, then $C$ is non-obstructed (i.e. the corresponding point of the Hilbert scheme is smooth). 
Proof: The case $k=2$ follows from Proposition 2.4, the case $k=1$ from [EF, Proposition 3.5] and the casc $k=0$ from [El].

Definition 2.6. A curve $C \subset \mathrm{P}^{3}$ is said to be quasi-complete intersection (bricfly q.c.i.) if there cxists a surjection $\Theta_{i=1}^{3} \mathcal{O}_{\mathrm{P}^{3}}\left(-a_{i}\right) \rightarrow I_{C} \rightarrow 0$ for some integers $a_{1} \leq a_{2} \leq a_{3}$.

Remark 2.6.1. This definition is equivalent to saying that there are homogeneous elements $f_{1}, f_{2}, f_{3} \in I(X)$ of degrees $a_{1}, a_{2}, a_{3}$, respectively, such that $I(X) /\left(f_{1}, f_{2}, f_{3}\right)$ is a graded $S$-module of finite length.

Remark 2.6.2. Let $X$ be a curve in $\mathbf{P}^{3}$. If $X$ is the zero scheme of a section of a rank 2 vector bundle on $\mathbf{P}^{3}$ and $Z$ is linked to $X$ then $Z$ is q.c.i. Conversely, if $Z$ is q.c.i. of threc surfaces of degrees $a_{1} \leq a_{2} \leq a_{3}$ and $X$ is linked to $Z$ by mears of two surfaces of degrees $a_{i}$ and $a_{j}$, respectively, then $X$ is the zero scheme of a section of a rank 2 vector bundle on $\mathbf{P}^{3}$.

Proposition 2.7. Let $Y \subset \mathbf{P}^{3}$ be an integral 2-Buchsbaum curve q.c.i. of three surfaces of degrees $9 \leq a_{1} \leq a_{2} \leq a_{3}$. Then, $Y$ is nonobstructed.

Remark. See [Mi], for the case of integral, 1-Buchsbaum, quasicomplete intersection space curves.

Proof: By definition there exists a surjection $\oplus_{i=1}^{3} \mathcal{O}_{\mathrm{P}^{3}}\left(-a_{i}\right) \rightarrow I_{Y} \rightarrow$ 0 . Set $F:=\operatorname{Ker}\left(\Theta_{i=1}^{3} \mathcal{O}_{\mathrm{p}^{3}}\left(-a_{i}\right) \rightarrow I_{Y}\right)$ and $E:=F\left(\left(a_{1}+a_{2}+a_{3}\right) / 2\right)$. By Proposition 1.3, $E$ is a 2 -Buchsbaum stable rank 2 vector bundle on $\mathbf{P}^{3}$ witl Chern classes $c_{1}=0, c_{2}=2$. Now, we link $Y$ to an irreducible curve $X$ by means of two surfaces of degrecs $a_{1}$ and $a_{2}$, respectively. By [PS, Proposition 2.5], the ideal sheaf $I_{X}$ of $X$ has a locally free resolution of the following type:

$$
0 \longrightarrow \mathcal{O}\left(a_{3}-a_{2}-a_{1}\right) \longrightarrow E\left(\left(a_{3}-a_{2}-a_{1}\right) / 2\right) \longrightarrow I_{X} \longrightarrow 0
$$

and by propositions 2.2 and $2.4 X$ is a non-obstructed, maximal rank space curve. Moreover, the hypothesis on $a_{i}$ 's and Proposition 1.4 imply that $H^{1} I_{X}\left(a_{2}-4\right)=H^{1} I_{X}\left(a_{1}-4\right)=0$. Thus, $Y$ is non-obstructed [K2, Corollary 3.1.0]. 


\section{Comments and Questions}

If we try to generalize the results of section 2 to higher values of $k$, we immediately encounter difficulties of various kinds, to be pointed out presently.

First of all, note that all subcanonical, 0-Buchsbaum curves are in the liaison class of a line; all subcanonical, 1-Buchsbaum curves are in the liaison class of the disjoint union of two lines; and all subcanonical, 2Buchsbaum curves are in the liaison class of the disjoint union of three lines. Thus, it is natural to ask if all subcanonical, $k$-Buchsbaum curves $(k>2)$ are in the liaison class of $\gamma=\gamma(k)$ lines or, at least, if all subcanonical, $k$-Buchsbaum curves $(k>2)$ are in the same liaison class. The answer, in general, is no. For instance:

Example 3.1. Take $Y_{1}$ the disjoint union of 4 lines, $Y_{2}$ the disjoint union of 5 lines: $Y_{3}$ a general, irreducible, smooth, elliptic curve of degree 7 and $Y_{4}$ a gencral, irreducible, smooth, elliptic curve of degree 8. $Y_{1}$, $Y_{2}, Y_{3}$ and $Y_{4}$ are subcanonical and it is not difficult to see that they are 3-Buchsbaum. However, computing their Hartshorne-Rao modules we easily get that they belong to four different liaison classes.

In section 2, we prove that subcanonical, $k$-Buchsbaum curves, $k \leq 2$, are non-obstructed. We wonder if the hypothesis $k \leq 2$ can be avoid. To be more precise, we suggest the following problems:

3.2.1. To characterize non-obstructed subcanonical space curves, and

3.2.2. To characterize non-obstrucled quesi-complete intersection space curves.

In particular,

3.3.1. Are subcamonical space curves of maximal rank non-obstructed?

3.3.2. Are quais-complete intersection space curves of maximal rank non-obstructed?

\section{References}

[BKM] G. Bolondi, J. O. Kleppe and R. M. Miró-Roig, Maximal rank curves and singular points of the Hibert schemme, Comp. Math. 77 (1991), 269-291.

[CV] L. Chian'ini and P. Vadabrega, Subcanonical curves and complete intersections in projective 3-spaces, Ann. Mat. Pura Appl. CXXXVIII (1984), 309-330. 
[E] Pri. Elbia, Ordres et cohomology des fibrés de rang delix sur l'espace projectif, Preprint (Nice) (1987).

[E1] PH. ElLLIA, D'autres composantes non-reduites de Hib $\mathbf{P}^{3}$, Math. Ann. 277 (1987), 433-446.

[EF] Ph. Ellia and M. Fiorentini, Quelques remarques sur les courbes arithmetiquenent Buchsbaurn de l'espace projectif, Ann. Univ. Ferrara XXXII (1987), 89-11.

[Ell] G. Ellingsrud, Sur le schema de Hilbert des variétés de codimension 2 dans $\mathbf{P}^{c}$ à cone Cohen-Macaulay, Ann. Sc. Ec- Norm. Sup) 2 (1975), 423-432.

[G] A. Grothfindeck, "Les schemas de Hilbert," Sem. Bourbaki 221, 1960 .

[H] R. HaRTshorne, Stable vector bundles of rank 2 on $\mathbf{P}^{3}$ : Math. Ann. 238 (1978), 229. 280.

[I] M. IDA, On the collonology of generic union of lines in $\mathbf{P}^{3}$, These (Nicc) (1986).

[K] J. O. KuEPPE, Deformalions of graded algebras, Math. Scand. 45 (1979), 205-231.

[Kl] J. O. KLEPPE, The Hilbert-flag scheme, its properties and its connection with the Hilbert scheme, Ph. Thesis (1981).

[K2] J. O. KLISP'”, "Liaison of families of subschemes in $\mathbf{P}^{n}$," LNM 1389, 1989, pp. 128-173.

[K3] J. O. KLLPPE, "Non-reduced compontents of the Hilbert scheme of smooth space curves," LNM 1266, 1987, pp. 181-207.

[K4] J. O. KLEPPE, On the existence of nice components components in the Hilbert scheme $H(d, g)$ of smooth connected space curves, Preprint (1990).

[M] D. Mumford, Further pathologies in Algebraic Geometry, Anter. J. Math. 89 (1962), 642-648.

[Mi] R. M. Miró-Rolg, Some remarks on Quasi-Complete Intersection Space Curves, Ann. Univ. Ferrara XXXIV (1988), 237-245.

[MM] J. Migliore and R. M. Miró-Roig, On k-Buchsbaum curves in $\mathbf{P}^{3}$, Comm. in Alg. 18 (1990), 2403-2422.

[S] E. Sernesi, Un esempio di curva ostruita in $\mathbf{P}^{3}$, Sem. variabili complesse (1981), 223-231, Bologna. 
[S1] E. SERnest, Topics on families of projective schemes, Queen's papers in Pure and Applied Math. 74 (1986).

[W] C. Walter, Some examples of obstructed curves in $\mathbf{P}^{3}$, Preprint, (1989).

Departament d'Algebra i Geometria

Current address:

Facultat de Matemàtiques

Departamento de Matomélicas

Universitat de Barcelona

Facultad de Ciencias

Gran Via 585

E- -08007 Barcclona

Universidad de Zaragoza

E-50009 Zaragoza

SPAIN

SPAIN

Rebut el 10 de Setembre de 1991 